\title{
Bilateral Repetitive Transcranial Magnetic Stimulation for Auditory Hallucinations in Patients with Schizophrenia: A Randomized Controlled, Cross-over Study
}

\author{
Eun-Ji Kim1, Seonguk Yeo', Inho Hwang, Jong-II Park', Yin Cui ${ }^{2,3}$, Hong-Mei Jin ${ }^{2,3}$, Hyung Tae Kim, Tae-Young Hwang ${ }^{4}$, \\ Young-Chul Chung ${ }^{1,2,3}$ \\ ${ }^{1}$ Department of Psychiatry, Chonbuk National University Hospital, ${ }^{2}$ Department of Psychiatry, Chonbuk National University Medical School, \\ ${ }^{3}$ Research Institute of Clinical Medicine of Chonbuk National University-Biomedical Research Institute of Chonbuk National University \\ Hospital, Jeonju, ${ }^{4}$ Department of Psychiatry, Chonbuk Provincial Maeumsarang Hospital, Wanju, Korea
}

\begin{abstract}
Objective: A randomized double-blind cross-over trial was conducted in patients with persistent auditory hallucinations (AHs) to investigate whether bilateral repetitive transcranial magnetic stimulation (rTMS) at the temporoparietal area or Broca's area is more effective at high- or low-frequencies compared to a sham condition.

Methods: Twenty three patients with persistent AHs who remained stable on the same medication for 2 months were enrolled. They were randomized to one of four conditions: low-frequency $(1 \mathrm{~Hz})-r T M S$ to the temporoparietal area $(\mathrm{L}-\mathrm{TP})$, high-frequency $(20 \mathrm{~Hz})-r T M S$ to the temporoparietal area $(\mathrm{H}-\mathrm{TP})$, high-frequency $(20 \mathrm{~Hz})-r T M S$ to Broca's area $(\mathrm{H}-\mathrm{B})$, or sham.

Results: All the four rTMS conditions resulted in significant decrease in the scores under the auditory hallucination rating scale and hallucination change scale over time. However, there were no significant treatment effects or interaction between time and treatment, suggesting no superior effects of the new paradigms over the sham condition.

Conclusion: Our findings suggest that bilateral rTMS at the temporoparietal area or Broca's area with high-or low-frequency does not produce superior effects in reducing AHs compared to sham stimulation.
\end{abstract}

KEY WORDS: Auditory hallucinations; Repetitive transcranial magnetic stimulation; Broca area.

\section{INTRODUCTION}

According to the Diagnostic and Statistical Manual of Mental Disorders 4th edition, text revision (DSM-IV-TR), ${ }^{1)}$ hallucination is "a sensory perception that has a compelling sense of reality of a true perception, but occurs without external stimulation of the relevant sensory organ." Auditory hallucinations (AHs) of a conversational or commanding nature are classified as first-rank symptoms of schizophrenia. AHs are found most often in patients with schizophrenia, with a prevalence of $75 \%$ in that population. ${ }^{2)}$ Moreover, $25-50 \%$ continue to experience them despite medication. ${ }^{3,4)}$ The experience of voices can impact on quality of life, self-esteem, anxiety, depression, suicide attempts, and cognitive function. ${ }^{5-7)}$

Repetitive transcranial magnetic stimulation (rTMS)

\footnotetext{
Received: May 9, 2014 / Revised: September 6, 2014

Accepted: September 28, 2014

Address for correspondence: Young-Chul Chung, MD, PhD Department of Psychiatry, Chonbuk National University Medical School, 20 Geonji-ro, Deokjin-gu, Jeonju 561-712, Korea Tel: +82-63-250-1390, Fax: +82-63-275-3157 E-mail: chungyc@jbnu.ac.kr
}

has recently been investigated as a potential new treatment for patients with treatment-resistant AHs. Low-frequency rTMS $(\leq 1 \mathrm{~Hz})$ has an inhibitory effect on motor cortical excitability in healthy individuals. ${ }^{8,9)}$ High-frequency rTMS $(>1 \mathrm{~Hz})$ reduces intracortical inhibition in healthy individuals ${ }^{10)}$ and promotes long-term neuroplasticity. ${ }^{11)}$ Since the first successful report by Hoffman et al.,${ }^{12)}$ many trials with different paradigms have yielded various results. Two recent reviews concluded that rTMS applied at the left temporoparietal area with a frequency of $1 \mathrm{~Hz}$ yielded a moderate mean-weighted effect size, ranging from 0.44 to 0.63 , indicating superiority of this paradigm; however, the results for other rTMS paradigms are disappointing. ${ }^{13,14)}$ Nevertheless, it should be noted that only a few investigations with other rTMS paradigms have been carried out. Critical questions with regard to other paradigms are as follows. First is whether bilateral rTMS is more effective than unilateral rTMS. Most trials have involved the administration of rTMS to the left temporoparietal cortex, but the relative importance of left or right hemispheric stimulation has only been minimally explored. There is some evidence to show that the pathol-

(c) This is an Open-Access article distributed under the terms of the Creative Commons Attribution Non-Commercial License (http://creativecommons.org/licenses/by-nc/3.0) which permits unrestricted non-commercial use, distribution, and reproduction in any medium, provided the original work is properly cited. 
ogy of AHs involves not only the left, but also the right hemisphere. AHs were associated with activation in the inferior frontal/insular, anterior cingulate, and bilateral temporal cortex (with greater responses on the right), the right thalamus and inferior colliculus, and the left hippocampus and parahippocampal cortex. ${ }^{15)}$ Hence, we assumed that bilateral rTMS would be more effective than unilateral rTMS. Second, although the effect size at other brain regions was lower $(0.33)$ compared to that of the left temporoparietal area $(0.44),{ }^{13)}$ should trials targeting other brain areas be further investigated. Neuroimaging studies of patients with AHs have identified activation in various brain regions other than temporoparietal area such as the anterior cingulate, ${ }^{15,16)}$ Broca's area, ${ }^{17,18)}$ hippocampus, and parahippocampal gyrus, ${ }^{19)}$ and primary auditory cortex. ${ }^{20)}$ Third, are there subtypes of patients with AHs or specific brain areas where high-frequency rTMS exerts superior effects? Two studies have reported positive results by delivering high-frequency rTMS at the posterior part of the left superior temporal sulcus or left temporoparietal cortex. $^{21,22)}$ From our clinical experiences, it seems that AHs generally tend to be reduced or suppressed when the patients talk to someone. Therefore, we hypothesized that the activation of Broca's area by high-frequency rTMS would reduce the severity of AHs. Fourth, will radically different paradigms such as theta burst stimulation (TBS), be more promising for the treatment of AHs? TBS was introduced as a patterned rTMS paradigm with brief stimulation sessions capable of generating lasting and reversible modulatory aftereffects on cortical motor neurophysiology. ${ }^{23)}$ Since continuous TBS, consisting of $50-\mathrm{Hz}$ bursts of three sub-threshold stimulations repeated at $5 \mathrm{~Hz}$, has inhibitory effects on corticospinal excitability, ${ }^{23)}$ it has been used to treat AHs, with clinical effects comparable to that produced by $1 \mathrm{~Hz}$ TMS. ${ }^{24)}$

The present study used a randomized double-blind cross-over design to assess the effects of rTMS to bilateral temporoparietal areas and Broca's area, compared to a sham-control.

\section{METHODS}

\section{Subjects}

Thirty patients with persistent AHs were recruited. Prior to participation, patients received written and oral information on the procedures and informed consent was obtained. Three patients refused to participate. Inclusion criteria were: 1) meeting the DSM-IV criteria for schizophrenia or schizoaffective disorder, 2) persistent AHs last- ing more than 6 months despite adequate trials of at least two antipsychotic agents (each for $>6$ weeks, with dose-equivalent to $\geq$ risperidone $6 \mathrm{mg}$ ), ${ }^{25)}$ and 3 ) no change of dosage of antipsychotic medication for 2 months before inclusion. Patients were required to remain on their psychotropic medication at steady dosages during the trial. The age range of the patients was 18 to 50 years. Exclusion criteria included a history of epileptic seizures, head trauma or mental retardation, or the presence of intracerebral or pacemaker implants, or pregnancy. Three patients were excluded for having mental retardation and one for a history of head trauma. Finally, twenty three patients were included and randomized, of which 22 completed the study. Among them, 21 were right-handed and one patient was ambidextrous according to the Edinburgh handedness inventory. ${ }^{26)}$ Eight patients were taking clozapine and continued to take it during treatment (Table 1).

Table 1. Demographic and clinical characteristics

\begin{tabular}{|c|c|}
\hline Characteristic & Data $(n=22)$ \\
\hline Sex (male/female) & $14(63.6) / 8(36.4)$ \\
\hline Age $(y)$ & $36.6 \pm 9.46$ \\
\hline Education (y) & $13.2 \pm 3.68$ \\
\hline \multicolumn{2}{|l|}{ Family status } \\
\hline Single & $18(81.8)$ \\
\hline Married & $3(13.6)$ \\
\hline Divorced & $1(4.5)$ \\
\hline \multicolumn{2}{|l|}{ Occupation } \\
\hline Student & $3(13.6)$ \\
\hline No & $17(77.3)$ \\
\hline Yes & $2(9.1)$ \\
\hline Familial psychiatric history (yes/no) & $2(9.1) / 20(90.9)$ \\
\hline Duration of illness (mon) & $130.86 \pm 101.70$ \\
\hline \multicolumn{2}{|l|}{ Handedness } \\
\hline Left-handed & $0(0.0)$ \\
\hline Right-handed & $21(96.4)$ \\
\hline Ambidextrous & $1(3.6)$ \\
\hline $\begin{array}{l}\text { Antipsychotic medication } \\
\text { (chlorpromazine equivalents) }\end{array}$ & $983.56 \pm 635.20$ \\
\hline \multicolumn{2}{|l|}{ Diagnosis } \\
\hline Schizophrenia, paranoid type & $20(90.9)$ \\
\hline Schizoaffective disorder & $1(4.5)$ \\
\hline Psychotic disorder NOS & $1(4.5)$ \\
\hline \multicolumn{2}{|l|}{ Rating scale scores } \\
\hline AHRS & $23.91 \pm 5.61$ \\
\hline \multicolumn{2}{|l|}{ PANSS } \\
\hline Positive & $19.64 \pm 3.85$ \\
\hline Negative & $19.27 \pm 3.06$ \\
\hline General & $39.18 \pm 6.01$ \\
\hline Total & $78.09 \pm 11.18$ \\
\hline CDSS & $5.05 \pm 5.07$ \\
\hline
\end{tabular}

Data are presented as number (\%) or mean \pm standard deviation. NOS, not otherwise specified; AHRS, Auditory Hallucination Rating Scale; CDSS, Calgary Depression Scale for Schizophrenia; PANSS, Positive and Negative Symptom Scale. 


\section{rTMS Protocols}

All patients received randomly scheduled treatment. A Latin square design with four modules (three active modules and one sham-control module) was used for randomization. Each module was performed twice a day (right and left sides, spaced by more than $3 \mathrm{~h}$ ) for three or five days. The interval between different modules was at least three days. This interval was chosen based on other studies where $2^{27)}$ or $4^{28)}$ days was used. The active modules were 1) low-frequency $(1 \mathrm{~Hz})-r T M S$ to the temporoparietal area (L-TP), 2) high-frequency (20 Hz)-rTMS to the temporoparietal area (H-TP), and 3) high-frequency $(20 \mathrm{~Hz})-\mathrm{rTMS}$ to Broca's area (H-B). The sites for the sham-control module were counterbalanced between the temporoparietal and Broca's areas and the stimulation coil was tilted at $45^{\circ}$ from a tangent to the head so that the front edge of the coil remained in contact with the head. This method reproduces sound and some somatic sensation (e.g., vibration and, at times, contraction of scalp muscles) that resemble active stimulation, while generating intracerebral voltages approximately $1 / 3$ of those of active TMS ${ }^{29,30)}$ High-frequency rTMS was delivered continuously over $5 \mathrm{~s}$ with 25 -s inter-train intervals, and each session of high-frequency rTMS lasted $10 \mathrm{~min}$ (twice a day for three days, total pulses $=12,000$ ). A low-frequency rTMS session lasted $20 \mathrm{~min}$ and the total pulses were the same as the high-frequency rTMS (twice a day for five days, total pulses $=12,000$ ). Stimulation was administered at $100 \%$ intensity of the motor threshold. Motor threshold was taken as the lowest TMS stimulus required to induce visible movement of the abductor pollicis brevis muscle in the relaxed hand on five out of ten occasions. The coil was held tangentially to the skull with the handle pointing dorsally. The temporoparietal stimulation was administered exactly halfway between the left temporal (T3) and left parietal (P3) sites, and between the right temporal (T4) and right parietal (P4) sites. Broca's region (left Brodmann areas 44 and 45) was localized as the crossing point between T3-Fz and F7-Cz. The right hemisphere homologue of Broca's area was localized as the crossing point between $\mathrm{T} 4-\mathrm{Fz}$ and $\mathrm{F} 8-\mathrm{Cz}$ according to the international 10-20 electroencephalogram (EEG) system. ${ }^{31)}$ The antipsychotic medications were continued without dose changes during the study.

\section{Clinical Evaluations}

Efficacy was measured using the Auditory Hallucination Rating Scale (AHRS) ${ }^{32)}$ and Hallucination Change
Scale (HCS) as the primary outcome measures. Each patient generated a narrative description of their AHs occurring for the 24-hour time period prior to initiation of the trial, which was set as their baseline score (for the HCS, scored as a 10). HCS and AHRS scores were ascertained again $24 \mathrm{~h}$ after stimulation on day 1, 3, or 5 (only low-frequency modules) of the trial immediately preceding the new session by requesting that the patient generate a new narrative description of their AHs occurring in the past 24 h. For the HCS, follow-up severity scores ranged from 0 , corresponding to no hallucinations, to a maximum score of 20 , corresponding to hallucinations twice as severe as baseline. Secondary outcome measures were the Positive and Negative Syndrome Scale (PANSS) ${ }^{33)}$ and Calgary Depression Scale for Schizophrenia (CDSS). ${ }^{34)}$ Measurements were conducted within a week before and after the rTMS treatment. The two raters (Yeo and Hwang) were blinded to rTMS modules, randomized, and inter-rater reliability was good with correlation coefficients of 0.72 . As the operators were not blinded to the protocol, they were not involved in the ratings. Adverse events were evaluated using the adverse events record form containing 9 items (headache, local pain, dizziness, memory difficulty, nausea, transient hearing changes, burns from scalp electrodes, meaningful mood changes, and other biological transient effects) which were the most commonly reported adverse reactions in previous rTMS studies. In addition, adverse events spontaneously reported were also recorded. Each evaluation was conducted right after each rTMS session, except for memory difficulty, which was measured on the subsequent days.

\section{Statistical Analysis}

Data obtained were analyzed with the Statistical Package for the Social Sciences (SPSS) version 12.0 for Windows (SPSS Inc., Chicago, IL, USA). A repeatedmeasures analysis of variance (ANOVA) was conducted on the AHRS and HCS scores to determine any differences from pre- to post-rTMS between the four rTMS conditions. Separately, paired-samples $t$ tests were conducted on the AHRS and HCS scores to assess significant changes from pre- to post-treatment for each of the four rTMS modules independently. Paired-samples $t$ tests were also conducted on the PANSS and CDSS scores to assess significant changes after completion of all the treatment schedules. Results were considered significant if $p<0.05$ (two-tailed). To compare the percentage of responders between the modules, Fisher's exact test was employed. Response to treatment was defined as an improvement in 
AHs, consisting of at least $20 \%$ reduction of the AHRS total scores from baseline.

\section{RESULTS}

\section{Treatment Efficacy}

The repeated-measures ANOVA revealed that there were no differences between the four rTMS modules. Although they all showed significant decreases of the AHRS and HCS scores over time $(p<0.001)$, there were no significant treatment effects or interaction effects between time and treatment (Table 2). Further analysis including the order of treatment as a covariate did not yield

Table 2. Comparison of the effects of four rTMS modules over time

\begin{tabular}{|c|c|c|c|c|c|}
\hline & \multirow[b]{2}{*}{ Pre-rTMS } & \multirow[b]{2}{*}{ Post-rTMS } & \multicolumn{3}{|c|}{$p$ value } \\
\hline & & & Time & Treatment & $\begin{array}{c}\text { Timex } \\
\text { Treatment }\end{array}$ \\
\hline \multicolumn{6}{|l|}{ AHRS } \\
\hline L-TP & $19.91 \pm 8.30$ & $17.82 \pm 8.96$ & 0.002 & 0.942 & 0.765 \\
\hline $\mathrm{H}-\mathrm{TP}$ & $20.41 \pm 7.04$ & $18.27 \pm 9.02$ & & & \\
\hline $\mathrm{H}-\mathrm{B}$ & $18.77 \pm 7.74$ & $17.95 \pm 8.44$ & & & \\
\hline Sham & $20.91 \pm 6.57$ & $18.55 \pm 8.62$ & & & \\
\hline \multicolumn{6}{|c|}{$\mathrm{HCS}$} \\
\hline L-TP & $8.50 \pm 2.56$ & $7.32 \pm 3.00$ & 0.001 & 0.985 & 0.491 \\
\hline $\mathrm{H}-\mathrm{TP}$ & $8.41 \pm 2.22$ & $7.77 \pm 2.43$ & & & \\
\hline $\mathrm{H}-\mathrm{B}$ & $8.36 \pm 2.44$ & $7.68 \pm 2.66$ & & & \\
\hline Sham & $8.00 \pm 2.41$ & $7.68 \pm 2.55$ & & & \\
\hline
\end{tabular}

Data are presented as mean \pm standard deviation.

rTMS, repetitive transcranial magnetic stimulation; AHRS, Auditory Hallucination Rating Scale; HCS, Hallucination Change Scale; L-TP, low-frequency temporoparietal area stimulation module: H-TP, high-frequency temporoparietal area stimulation module; $\mathrm{H}-\mathrm{B}$, high-frequency Broca's area stimulation module; Sham, Sham control module.

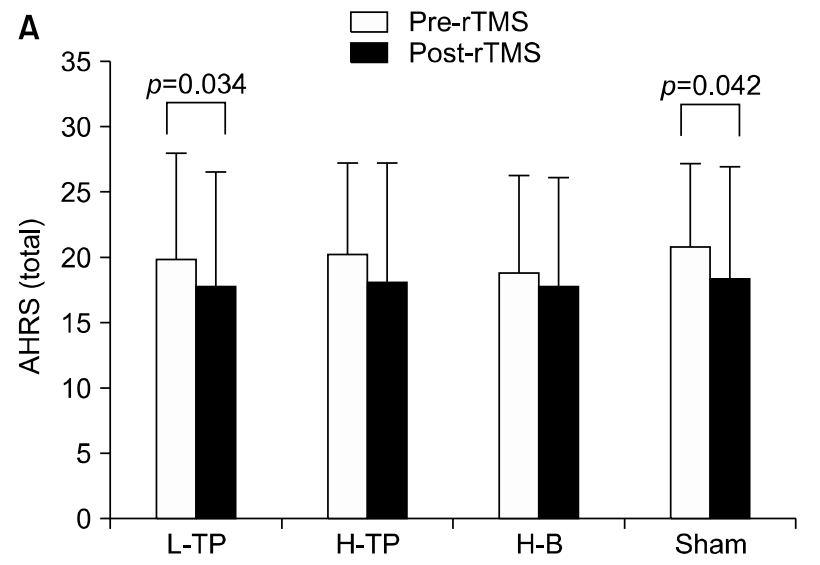

significant results. Separate paired $t$ tests in each module revealed that the AHRS and HCS scores decreased significantly in the L-TP module. However, there was also a significant change of the AHRS scores in the sham module. There was no significant improvement on the AHRS and HCS scores in the H-TP and H-B modules (Fig. 1). The PANSS positive, general, and total scores were significantly improved after the completion of treatment schedules but the PANSS negative score and CDSS score did not change significantly (Table 3 ).

In the L-TP and H-TP modules, six of 22 patients were responders $(27.3 \%)$, whereas two patients in the H-B module $(9.1 \%)$ and three patients in the sham module (13.1\%) were responders. Fisher test revealed no significant difference between the numbers of patients responding to each of the four rTMS modules.

\section{Adverse Effects}

Generally, the treatment was well tolerated and no serious adverse effects such as seizures were reported. Adverse effects reported during the treatment are outlined in Table 4. We counted the number of patients reporting adverse effects during each stimulation module. The most common adverse effect was local pain at the site of stimulation, especially in the H-B module, reported by seven patients (31.8\%). Other common adverse effects were dizziness and headache. One patient reported toothache on the same side as the stimulation site. In the sham module, only local pain and headache were reported. All these symptoms were transient and lasted only a few minutes after stimulation.

Fig. 1. The change of auditory hallucination rating scale (AHRS) scores (A) and hallucination change scale (HCS) scores (B) in the four repetitive transcranial magnetic stimulation (rTMS) modules.

L-TP, low-frequency temporoparietal area stimulation module; H-TP, high-frequency temporoparietal area stimulation module; H-B, high-frequency Broca's area stimulation module; Sham, sham control module. 
Table 3. Change in scores before and after the completion of treatment schedules

\begin{tabular}{lcrcc}
\hline & Pre-rTMS & Post-rTMS & $\dagger$ & $p$ value \\
\hline PANSS & & & & \\
Positive & $19.64 \pm 3.85$ & $17.77 \pm 2.54$ & 2.886 & 0.009 \\
Negative & $19.27 \pm 3.06$ & $18.55 \pm 2.92$ & 1.919 & 0.069 \\
General & $39.18 \pm 6.01$ & $38.09 \pm 4.87$ & 2.124 & 0.046 \\
Total & $78.09 \pm 11.18$ & $74.41 \pm 9.18$ & 2.858 & 0.009 \\
CDSS & $5.05 \pm 5.07$ & $4.55 \pm 5.40$ & 1.176 & 0.253 \\
\hline
\end{tabular}

Data are presented as mean \pm standard deviation.

rTMS, repetitive transcranial magnetic stimulation; PANSS, Positive and Negative Symptom Scale; CDSS, Calgary Depression Scale for Schizophrenia.

\section{DISCUSSION}

In spite of substantial evidence pointing to a moderate effect size of L-TP on the left side for reducing AHs, we assumed that different paradigms should be further examined in terms of bilaterality, sites, and frequency of stimulation. To test whether bilateral rTMS at the temporoparietal area or Broca's area with high- or low-frequency is more effective than the sham condition, we conducted a randomized double-blind cross-over design in patients with persistent AHs. Disappointingly, we observed no significant differences in the improvement of AHs between the four rTMS modules. In other words, the effects of different paradigms on the temporoparietal area or Broca's area were not superior compared to the sham condition.

rTMS was mostly applied to the left temporoparietal area. However, functional magnetic resonance imaging (fMRI) studies show that in approximately $50 \%$ of patients hallucinatory activation involves the right hemisphere. $^{35)}$ The right hemisphere is considered to be involved in the nonlinguistic aspects of language processing, such as emotional prosody and non-literal meaning comprehension. ${ }^{36)}$ Moreover, decreased language lateralization has been reported in schizophrenia, indicating an increase in language-related activity in the right hemisphere $^{37)}$ in these patients. This led to the hypothesis that when language activity is derived from an unusual site (i.e., from contralateral homologue areas in the right hemisphere), inhibition of language perception might be more prone to failure. This may make inner speech become perceived as AHs. ${ }^{35)}$ All these findings prompted us to assume that bilateral stimulation would be more effective in alleviating AHs as compared to unilateral stimulation. However, we did not include a control for bilaterality (i.e., unilateral stimulation); hence, our results only indicated that bilateral stimulation to either the temporoparietal area or Broca's area did not have superior effects compared to
Table 4. Adverse effects

\begin{tabular}{lcccc}
\hline & L-TP & H-TP & H-B & Sham \\
\hline Headache & 1 & 1 & 1 & 1 \\
Local pain & 5 & 5 & 7 & 4 \\
Dizziness & 2 & 1 & 3 & 0 \\
Memory difficulty & 0 & 0 & 0 & 0 \\
Nausea & 0 & 0 & 0 & 0 \\
Transient hearing changes & 0 & 0 & 0 & 0 \\
Burn & 0 & 0 & 0 & 0 \\
Meaningful mood change & 0 & 0 & 0 & 0 \\
Toothache & 1 & 1 & 1 & 0 \\
\hline
\end{tabular}

Data are given as number of patients.

L-TP, low-frequency temporoparietal area stimulation module; $\mathrm{H}$-TP, high-frequency temporoparietal area stimulation module; $\mathrm{H}-\mathrm{B}$, high-frequency Broca's area stimulation module; Sham, sham control module.

the sham stimulation condition. Similarly, one randomized controlled study reported that bilateral stimulation to the temporoparietal region was not as effective as unilateral stimulation in reducing hallucination frequency. ${ }^{38)}$

Broca's area is one of the main areas of the cerebral cortex responsible for producing language. McGuire et al. ${ }^{18)}$ first reported that blood flow in Broca's area was significantly greater during hallucinations than in a non-hallucinating state. This finding was considered as supporting evidence for the inner-speech model of AHs, especially generation abnormalities of inner speech. ${ }^{39)}$ However, several other imaging studies have failed to replicate the finding that inferior frontal gyrus (including Broca's area) activation is associated with the experience of AHs. ${ }^{19,40)}$ This may partly explain that only a few studies have been conducted to confirm the effects of rTMS on Broca's area in patients with AHs. Until now, two studies ${ }^{27,41)}$ have reported negative results when rTMS was delivered to Broca's area. It is, however, of note that most of the participants in the two studies had chronic schizophrenia, and low-frequency stimulation was used. Although we obtained similar negative results, this is the first trial using bilateral high-frequency stimulation targeting Broca's area and its right homologous area.

Typically, low frequency rTMS has been used in the treatment of AHs, based on the fact that low frequency rTMS produces an inhibitory effect when applied to the sensory motor cortex. ${ }^{42)}$ However, we were particularly interested in applying high-frequency stimulation given that it decreases metabolism in the target area, ${ }^{43)}$ suspends higher cognitive functions ${ }^{44)}$ such as speech production, ${ }^{45)}$ and improves pathological conditions including neuropathic pain and tinnitus. ${ }^{46)}$ Moreover, this method has an advantage in terms of reducing treatment duration, i.e., $\sim 2-3$ days compared to 2 weeks with low-frequency 
stimulation. Montagne-Larmurier et al. ${ }^{22)}$ reported that a significant reduction in global severity and frequency of AHs between baseline and post-treatment day 12 was observed in patients with AHs receiving high-frequency (20 $\mathrm{Hz}$ ) rTMS to the left temporoparietal cortex for 2 days. Unfortunately, we observed no significant effects of $\mathrm{H}-\mathrm{TP}$ compared to the sham condition. The discrepancy may be due to the methodological difference in localizing the stimulation site. They used fMRI to localize the posterior part of the left superior temporal sulcus whereas we simply used the 10-20 EEG system. It is worthnoting that no serious side effects, such as seizures, were observed with the high-frequency modules (H-TP and H-B). In general, high-frequency stimulation modules were well tolerated except for local pain most frequently noted in the H-B module (Table 4).

This study has several limitations. First, as the interval between the modules was usually 3-5 days, carryover effects may have come into play as a confounding factor. The long-term efficacy of rTMS is known to be over a number of weeks. ${ }^{47,48)}$ Nevertheless, as the present study was exploratory in nature, we shortened the interval with the concern that long interval would increase a drop-out rate. In addition, to extract this order effect, an ANCOVA was carried out. However, it made no difference, indicating a low likelihood of an order effect. Second, sham stimulation in this study was administered with active rTMS coils with their front edge touching the scalp at $45^{\circ}$. This procedure is not exempt from criticism. Indeed, in a double-blind controlled study in depressed patients, the authors failed to find a significant difference between real and sham TMS treatment, with the sham group demonstrating an improvement of about $25 \% .{ }^{49)}$ Using a sham coil at the same location, strength, and frequency could be more useful for comparing active rTMS and sham in future studies. Third, the efficacy of targeting Broca's area with rTMS may be questioned because the anterior part of the temporalis muscle lies above Broca's area, limiting conductivity of the rTMS. To avoid this problem, deep TMS capable of penetrating $\sim 4.5-5.5 \mathrm{~cm}$ into the brain could be considered. Fourth, the duration of illness of the participants was more than 10 years. Had we recruited patients with a more recent onset, the results may have been different. With these caveats in mind, our results should be interpreted cautiously. The strengths of our study are its double-blind randomized controlled design and it being the first attempt at targeting Broca's area with high-frequency stimulation.

In conclusion, our findings suggest that bilateral rTMS at the temporoparietal area or Broca's area with high- or low-frequency does not produce superior effects in reducing AHs compared to sham stimulation. Nevertheless, efforts searching for optimal conditions using different paradigms should be continued.

\section{Acknowledgments}

This work was supported by a grant from Kye-Nam, Kim Jae Jung Memorial Fund (Grant number 16) and Fund of Biomedical Research Institute, Chonbuk National University Hospital, Korea.

\section{REFERENCES}

1. American Psychiatric Association, Task force on DSM-IV. Diagnostic and statistical manual of mental disorders: DSM-IV-TR. 4th ed. Washington, DC: American Psychiatric Association; 2000.

2. Nayani TH, David AS. The auditory hallucination: a phenomenological survey. Psychol Med 1996;26:177-189.

3. Carter DM, Mackinnon A, Copolov DL. Patients' strategies for coping with auditory hallucinations. J Nerv Ment Dis 1996;184:159-164.

4. Pantelis C, Barnes TR. Drug strategies and treatmentresistant schizophrenia. Aust N Z J Psychiatry 1996;30:20-37.

5. Breier A, Schreiber JL, Dyer J, Pickar D. National Institute of Mental Health longitudinal study of chronic schizophrenia. Prognosis and predictors of outcome. Arch Gen Psychiatry 1991;48:239-246.

6. Harkavy-Friedman JM, Kimhy D, Nelson EA, Venarde DF, Malaspina D, Mann JJ. Suicide attempts in schizophrenia: the role of command auditory hallucinations for suicide. $J$ Clin Psychiatry 2003;64:871-874.

7. Lee SH, Choi BH, Song HS, Chung YC, Suh KY. Neurocognitive Function and clinical characteristic in schizophrenia patients with treatment-resistant auditory hallucination. J Korean Neuropsychiatr Assoc 2004;43:288-295.

8. Chen R, Classen J, Gerloff C, Celnik P, Wassermann EM, Hallett M, et al. Depression of motor cortex excitability by low-frequency transcranial magnetic stimulation. Neurology 1997;48:1398-1403.

9. Wassermann EM, Lisanby SH. Therapeutic application of repetitive transcranial magnetic stimulation: a review. Clin Neurophysiol 2001;112:1367-1377.

10. Ziemann U, Rothwell JC, Ridding MC. Interaction between intracortical inhibition and facilitation in human motor cortex. J Physiol 1996;496:873-881.

11. Esser SK, Huber R, Massimini M, Peterson MJ, Ferrarelli F, Tononi G. A direct demonstration of cortical LTP in humans: a combined TMS/EEG study. Brain Res Bull 2006;69:86-94.

12. Hoffman RE, Boutros NN, Berman RM, Roessler E, Belger A, Krystal JH, et al. Transcranial magnetic stimulation of left temporoparietal cortex in three patients reporting hallucinated "voices". Biol Psychiatry 1999;46:130-132.

13. Slotema CW, Aleman A, Daskalakis ZJ, Sommer IE. Metaanalysis of repetitive transcranial magnetic stimulation in the treatment of auditory verbal hallucinations: update and effects after one month. Schizophr Res 2012;142:40-45.

14. Slotema CW, Blom JD, van Lutterveld R, Hoek HW, Sommer IE. Review of the efficacy of transcranial magnetic 
stimulation for auditory verbal hallucinations. Biol Psychiatry 2014;76:101-110.

15. Shergill SS, Brammer MJ, Williams SC, Murray RM, McGuire PK. Mapping auditory hallucinations in schizophrenia using functional magnetic resonance imaging. Arch Gen Psychiatry 2000;57:1033-1038.

16. Suzuki M, Yuasa S, Minabe Y, Murata M, Kurachi M. Left superior temporal blood flow increases in schizophrenic and schizophreniform patients with auditory hallucination: a longitudinal case study using 123I-IMP SPECT. Eur Arch Psychiatry Clin Neurosci 1993;242:257-261.

17. Dierks T, Linden DE, Jandl M, Formisano E, Goebel R, Lanfermann $\mathrm{H}$, et al. Activation of Heschl's gyrus during auditory hallucinations. Neuron 1999;22:615-621.

18. McGuire PK, Shah GM, Murray RM. Increased blood flow in Broca's area during auditory hallucinations in schizophrenia. Lancet 1993;342:703-706.

19. Silbersweig DA, Stern E, Frith C, Cahill C, Holmes A, Grootoonk S, et al. A functional neuroanatomy of hallucinations in schizophrenia. Nature 1995;378:176-179.

20. van de Ven VG, Formisano E, Röder CH, Prvulovic D, Bittner RA, Dietz MG, et al. The spatiotemporal pattern of auditory cortical responses during verbal hallucinations. Neuroimage 2005;27:644-655.

21. Dollfus S, Larmurier-Montagne A, Razafimandimby A, Allio G, Membrey JM, Delcroix N, et al. Treatment of auditory hallucinations by combining high-frequency repetitive transcranial magnetic stimulation and functional magnetic resonance imaging. Schizophr Res 2008;102:348-351.

22. Montagne-Larmurier A, Etard O, Razafimandimby A, Morello R, Dollfus S. Two-day treatment of auditory hallucinations by high frequency rTMS guided by cerebral imaging: a 6 month follow-up pilot study. Schizophr Res 2009; 113:77-83.

23. Huang YZ, Edwards MJ, Rounis E, Bhatia KP, Rothwell JC. Theta burst stimulation of the human motor cortex. Neuron 2005:45:201-206.

24. Kindler J, Homan P, Flury R, Strik W, Dierks T, Hubl D. Theta burst transcranial magnetic stimulation for the treatment of auditory verbal hallucinations: results of a randomized controlled study. Psychiatry Res 2013;209:114-117.

25. Kubera KM, Sambataro F, Vasic N, Wolf ND, Frasch K, Hirjak D, et al. Source-based morphometry of gray matter volume in patients with schizophrenia who have persistent auditory verbal hallucinations. Prog Neuropsychopharmacol Biol Psychiatry 2014;50:102-109.

26. Oldfield RC. The assessment and analysis of handedness: the Edinburgh inventory. Neuropsychologia 1971;9:97-113.

27. Schönfeldt-Lecuona $C$, Grön $G$, Walter $H$, Büchler N, Wunderlich A, Spitzer M, et al. Stereotaxic rTMS for the treatment of auditory hallucinations in schizophrenia. Neuroreport 2004;15:1669-1673.

28. Loo CK, Sainsbury K, Mitchell P, Hadzi-Pavlovic D, Sachdev PS. A sham-controlled trial of left and right temporal rTMS for the treatment of auditory hallucinations. Psychol Med 2010;40:541-546.

29. Lisanby SH, Gutman D, Luber B, Schroeder C, Sackeim HA. Sham TMS: intracerebral measurement of the induced electrical field and the induction of motor-evoked potentials. Biol Psychiatry 2001;49:460-463.

30. Loo CK, Taylor JL, Gandevia SC, McDarmont BN, Mitchell PB, Sachdev PS. Transcranial magnetic stimulation (TMS) in controlled treatment studies: are some "sham" forms active? Biol Psychiatry 2000;47:325-331.

31. Friederici AD, Hahne A, von Cramon DY. First-pass versus second-pass parsing processes in a Wernicke's and a Broca's aphasic: electrophysiological evidence for a double dissociation. Brain Lang 1998;62:311-341.

32. Hoffman RE, Hawkins KA, Gueorguieva R, Boutros NN, Rachid F, Carroll K, et al. Transcranial magnetic stimulation of left temporoparietal cortex and medication-resistant auditory hallucinations. Arch Gen Psychiatry 2003;60:49-56.

33. Kay SR, Fiszbein A, Opler LA. The positive and negative syndrome scale (PANSS) for schizophrenia. Schizophr Bull 1987;13:261-276.

34. Addington D, Addington J, Maticka-Tyndale E. Assessing depression in schizophrenia: the Calgary Depression Scale. Br J Psychiatry Suppl 1993;(22):39-44.

35. Sommer IE, Aleman A, Kahn RS. Left with the voices or hearing right? Lateralization of auditory verbal hallucinations in schizophrenia. J Psychiatry Neurosci 2003;28:217-218.

36. Mitchell RL, Crow TJ. Right hemisphere language functions and schizophrenia: the forgotten hemisphere? Brain 2005;128:963-978.

37. Sommer IE, Ramsey NF, Kahn RS. Language lateralization in schizophrenia, an fMRI study. Schizophr Res 2001;52:57-67.

38. Vercammen A, Knegtering H, Bruggeman R, Westenbroek $\mathrm{HM}$, Jenner JA, Slooff CJ, et al. Effects of bilateral repetitive transcranial magnetic stimulation on treatment resistant auditory-verbal hallucinations in schizophrenia: a randomized controlled trial. Schizophr Res 2009;114:172-179.

39. Allen P, Aleman A, McGuire PK. Inner speech models of auditory verbal hallucinations: evidence from behavioural and neuroimaging studies. Int Rev Psychiatry 2007;19:407-415.

40. Lennox BR, Park SB, Medley I, Morris PG, Jones PB. The functional anatomy of auditory hallucinations in schizophrenia. Psychiatry Res 2000;100:13-20.

41. Hoffman RE, Hampson M, Wu K, Anderson AW, Gore JC, Buchanan RJ, et al. Probing the pathophysiology of auditory/ verbal hallucinations by combining functional magnetic resonance imaging and transcranial magnetic stimulation. Cereb Cortex 2007; 17:2733-2743.

42. Pascual-Leone A, Tormos JM, Keenan J, Tarazona F, Cañete C, Catalá MD. Study and modulation of human cortical excitability with transcranial magnetic stimulation. J Clin Neurophysiol 1998; 15:333-343.

43. Paus T, Jech R, Thompson CJ, Comeau R, Peters T, Evans AC. Dose-dependent reduction of cerebral blood flow during rapid-rate transcranial magnetic stimulation of the human sensorimotor cortex. J Neurophysiol 1998;79:1102-1107.

44. Hallett M. Transcranial magnetic stimulation and the human brain. Nature 2000;406:147-150.

45. Devlin JT, Watkins KE. Stimulating language: insights from TMS. Brain 2007;130:610-622.

46. Ridding MC, Rothwell JC. Is there a future for therapeutic use of transcranial magnetic stimulation? Nat Rev Neurosci 2007:8:559-567.

47. Hoffman RE, Gueorguieva R, Hawkins KA, Varanko M, Boutros NN, Wu YT, et al. Temporoparietal transcranial magnetic stimulation for auditory hallucinations: safety, efficacy and moderators in a fifty patient sample. Biol Psychiatry 2005;58:97-104.

48. Chibbaro G, Daniele M, Alagona G, Di Pasquale C, Cannavò $\mathrm{M}$, Rapisarda V, et al. Repetitive transcranial magnetic stimulation in schizophrenic patients reporting auditory hallucinations. Neurosci Lett 2005;383:54-57.

49. Loo C, Mitchell P, Sachdev P, McDarmont B, Parker G, Gandevia S. Double-blind controlled investigation of transcranial magnetic stimulation for the treatment of resistant major depression. Am J Psychiatry 1999;156:946-948. 